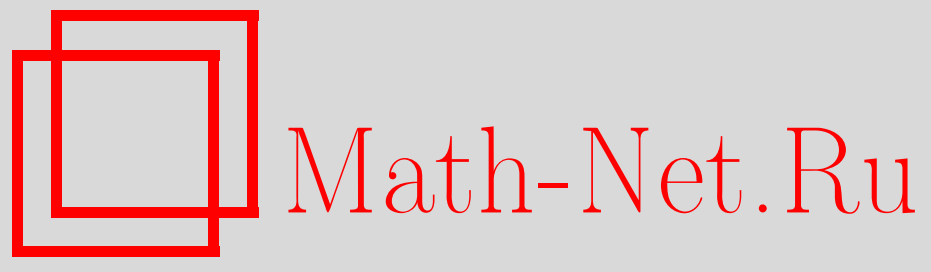

Э. Превиато, В. З. Энольский, Ультраэллиптические солитоны, УМН, 2007, том 62, выпуск 4, 173-174

DOI: https://doi.org/10.4213/rm6812

Использование Общероссийского математического портала Math-Net.Ru подразумевает, что вы прочитали и согласны с пользовательским соглашением http://www . mathnet.ru/rus/agreement

Параметры загрузки:

IP: 3.85 .183 .62

26 апреля 2023 г., $16: 25: 42$

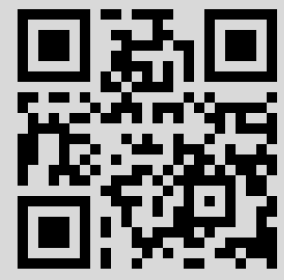




\section{Ультраэллиптические солитоны}

\section{Э. Превиато, В. З. Энольский}

В этом сообщении описывается обобщение "эллиптических солитонов", т. е. двоякопериодических по первому потоку решений КП-иерархии, названных так Ж.-Л. Вердье [1] и классифицированных И. М. Кричевером [2]. Мы определяем "ультраэллиптические" решения (т. е. периодические на якобиане рода 2). Мы вводим следующий анзац:

$$
\mathscr{U}\left(t_{1}, \ldots, t_{g}\right)=2 \sum_{i=1}^{N} \wp_{22}\left(\boldsymbol{u}-\boldsymbol{X}_{i}\right), \quad \boldsymbol{u}=\left(\begin{array}{c}
t \\
x
\end{array}\right), \quad \boldsymbol{X}=\left(\begin{array}{c}
\mathfrak{T}_{i}\left(t_{3}, \ldots, t_{g}\right) \\
\mathfrak{X}_{i}\left(t_{3}, \ldots, t_{g}\right)
\end{array}\right)
$$

(при возможном переупорядочении времен $t_{k}$ ), где $\wp_{22}$ - аналог рода 2 для функции Вейерштрасса ю, обобщающий анзац Кричевера. Мы вернемся к рассмотрению "прямой" спектральной задачи, классифицирующей ультраэллиптические солитоны, в одной из последующих публикаций.

ОСНовная теОРемА. Пусть $V_{g}$ - алгебраическая кривая рода $g>2$, и пусть $\left(U_{1 k}, \ldots, U_{g k}\right)^{T}-k$-й столбец, обратной матрицы $2 \omega^{-1}$ периодов нормированных голоморфных дифференциалов. g-зонное решение уравнения КП сводится к сумме ультраэллиптических солитонов, если: (i) $V_{g} \rightarrow V_{2}$ есть накрытие, (ii) в базисе гомологий, в котором $\tau$-матрица имеет вид $\left(\begin{array}{cc}\tau_{2} & Q \\ Q^{T} & \tau_{g-2}\end{array}\right)$, где $Q$-рачиональная матрица размера $2 \times(g-2)$, выполняются следующие равенства:

$$
U_{31}=\cdots=U_{g 1}=0, \quad U_{32}=\cdots=U_{g 2}=0 .
$$

Мы доказываем эту теорему, используя классическую теорию редукции матриц периодов [3] и геометрическую конструкцию подходящим образом нормированного базиса гомологий кривой, см. [4]. Мы используем затем ю-функции Клейна (определения см. в [5]) гиперэллиптической кривой рода $2, V_{2} \ni(x, y): y^{2}=4 x^{5}+\sum_{i=0}^{3} \lambda_{i} x^{i}$, $\lambda_{j} \in \mathbb{C}$, и разложения $\sigma$-функции при $\boldsymbol{u}=(0,0)$ (недавно В. М. Бухштабер и Д. В. Лейкин [6] предъявили рекурсивные формулы для коэффициентов разложения, определив операторную алгебру Ли, зануляющую $\sigma$-функцию, см. также [7]), где получены алгебро-геометрические решения уравнения КП $\mathscr{U}_{y y}=\left(\mathscr{U}_{t}-6 \mathscr{U}_{x} \mathscr{U}_{x}-\mathscr{U}_{x x x}\right)_{x}$, определяемые по формуле [8]

$$
\mathscr{U}(x, y, t)=-2 \frac{\partial^{2}}{\partial x^{2}} \log \theta\left(\boldsymbol{U}_{1} x+\boldsymbol{U}_{2} y+\boldsymbol{U}_{3} t ; \tau\right)+C .
$$

Для демонстрации существования ультраэллиптических солитонов рассмотрим гиперэллиптическую кривую рода три, $V_{3}=(x, y)[7]:$

$$
y^{2}=\prod_{k=1}^{4}\left(x^{2}-a_{k}^{2}\right) \equiv \Lambda_{8} x^{8}+\Lambda_{6} x^{6}+\cdots+\Lambda_{0} .
$$

Кривая допускает инволюцию $(x, y) \rightarrow(-x, y)$, накрывая при этом гиперэллиптическую кривую рода два $V_{2}=(z, w)$ и эллиптическую кривую $V_{1}=(Z, W)$, задаваемые уравнениями

$$
w^{2}=4 z \prod_{k=1}^{4}\left(z-a_{k}^{2}\right), \quad W^{2}=4 \prod_{k=1}^{4}\left(Z-a_{k}^{2}\right) .
$$

Голоморфные дифференциалы на $V_{3}$ сводятся подстановками $x^{2}=z$ и $x^{2}=Z$ к голоморфным дифференциалам на $V_{2}$ и $V_{1}$ (заметим, что временами потоков, соответствующих ультраэллиптической функции, являются $t_{1}$ и $\left.t_{3}\right)$ :

$$
\frac{\mathrm{d} x}{y}=\frac{\mathrm{d} z}{w}, \quad \frac{x^{2} \mathrm{~d} x}{y}=\frac{z \mathrm{~d} z}{w} \quad \text { и } \quad \frac{x \mathrm{~d} x}{y}=\frac{\mathrm{d} Z}{W} .
$$


Рассмотрим уравнение

$$
\mathfrak{P}_{3,3,3,3}=6 \mathfrak{P}_{3,3}^{2}-\frac{1}{2} \Lambda_{4} \Lambda_{8}+\Lambda_{8}\left(4 \mathfrak{P}_{1,3}-3 \mathfrak{P}_{2,2}\right)+\Lambda_{6} \mathfrak{P}_{3,3},
$$

ассоциированное с кривой (1), где символ $\mathfrak{P}_{i, j}$ введен для того, чтобы отличить функцию Клейна ю кривой (1) от функции Клейна рода 2. Для получения стандартного уравнения КП на функцию $\mathscr{U}(x, y, t)$ следует продифференцировать (2) дважды по $u_{3}$ и положить $2 \mathfrak{P}_{3,3}(t, y, x)=\mathscr{U}(x, y, t)$ и $\Lambda_{0}=0$.

Введем анзац

$$
\mathfrak{P}_{3,3}(t, y, x)=\sum_{i=1}^{N} \wp_{2,2}\left(\boldsymbol{u}-\boldsymbol{X}_{i}\right), \quad \boldsymbol{u}=\left(\begin{array}{c}
t \\
x
\end{array}\right), \quad \boldsymbol{X}_{i}=\left(\begin{array}{c}
F_{i}(y) \\
G_{i}(y)
\end{array}\right),
$$

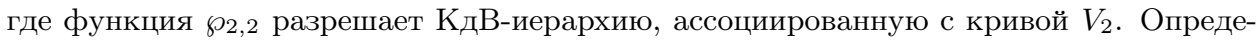
лим неизвестные функции $F_{i}(y), G_{i}(y)$, подставляя (3) в (2) (заметим, что $N=2$ ) и полагая $\boldsymbol{u} \rightarrow \boldsymbol{X}_{i}$. Мы используем разложение $\sigma$-функции кривой $V_{2}$ и получаем уравнение:

$$
\begin{gathered}
\left(\frac{\mathrm{d} F_{1}(y)}{\mathrm{d} y}\right)^{2}=4 \wp_{2,2}\left(\boldsymbol{X}_{1}-\boldsymbol{X}_{2}\right)-\frac{4}{3} \Lambda_{4}, \quad \frac{\mathrm{d}^{2} G_{1}(y)}{\mathrm{d} y^{2}}=-14 \wp_{2,2,2}\left(\boldsymbol{X}_{1}-\boldsymbol{X}_{2}\right), \\
\frac{\mathrm{d} G_{1}(y)}{\mathrm{d} y}\left(\frac{\mathrm{d} G_{1}(y)}{\mathrm{d} y}+2 \frac{\mathrm{d} F_{1}(y)}{\mathrm{d} y}\right)=0 .
\end{gathered}
$$

Нетривиальные решения этой системы можно получить, положив

$$
\frac{\mathrm{d} G_{k}(y)}{\mathrm{d} y}+2 \frac{\mathrm{d} F_{k}(y)}{\mathrm{d} y}=0, \quad k=1,2, \quad \frac{\mathrm{d} F_{1}(y)}{\mathrm{d} y}=-\frac{\mathrm{d} F_{2}(y)}{\mathrm{d} y} .
$$

Последние равенства приводят к геометрическому условию (выделяющему локус)

$$
\wp_{2,2,2}\left(\boldsymbol{X}_{1}-\boldsymbol{X}_{2}\right)+4 \wp_{1,2,2}\left(\boldsymbol{X}_{1}-\boldsymbol{X}_{2}\right)=0 .
$$

Мы полагаем, что этот локус совпадает с КдВ-локусом при дальнейших редукциях, но заметим, что, в отличие от случая эллиптических солитонов, двухчастичный локус может оказаться непустым.

\section{Список литературы}

[1] J. L. Verdier, Algebraic analysis, Vol. 2, Academic Press, Boston, MA, 1988, 901-910. [2] И. М. Кричевер, Функи. анализ и его прилож., 14:4 (1980), 45-54. [3] H. Poincaré, Bull. Soc. Math. France, 12 (1884), 124-143. [4] H. H. Martens, Curves, Jacobians, and abelian varieties (Amherst, MA, USA, 1990), Contemp. Math., 136, Amer. Math. Soc., Providence, RI, 1992, 287-296. [5] V. M. Buchstaber, V. Z. Enolskii, D. V. Leykin, Solitons, geometry and topology: on the crossroad, Amer. Math. Soc. Transl. Ser. 2, 179, eds. V. M. Buchstaber, S. P. Novikov, Amer. Math. Soc., Providence, RI, 1997, 1-33.[6] В.М.Бухштабер, Д. В. Лейкин, Функи. анализ и его прилож., 36:4 (2002), 18-34. [7] H. F. Baker, An introduction to the theory of multiply-periodic functions, Cambridge Univ. Press, Cambridge, 1907. [8] И. М. Кричевер, Функи. анализ и его прилож., 11:1 (1977), 15-31.

\section{Э. Превиато (Е. Previato)}

Boston University, USA

E-mail: ep@bu.edu

В. З. Энольский (V.Z. Ènol'skii)

Институт магнетизма НАН Украины

E-mail: vze@ma.hw.ac.uk
Представлено И. М. Кричевером Принято редколлегией 06.04.2007 